\title{
Chromosomal locations of the maize (Zea mays L.) HtP and $r$ genes that confer resistance to Exserohilum turcicum
}

\author{
Juliana Bernardi Ogliari ${ }^{1}$, Marco Antônio Guimarães ${ }^{2}$ and Luis Eduardo Aranha Camargo ${ }^{3}$ \\ ${ }^{1}$ Departamento de Fitotecnia, Centro de Ciências Agrárias, Universidade Federal de Santa Catarina, \\ Florianópolis, SC, Brazil. \\ ${ }^{2}$ Monsanto do Brasil Ltda, Uberlândia, MG, Brazil. \\ ${ }^{3}$ Setor de Fitopatologia, Escola Superior de Agricultura Luiz de Queiroz, Universidade de São Paulo, \\ Piracicaba, SP, Brazil.
}

\begin{abstract}
We used 125 microsatellite markers to genotype the maize (Zea mays L.) near isogenic lines (NIL) L30 $0^{H t P H t P R \text { tRt }}$ and $\mathrm{L} 30^{\text {htphtpRtRt }}$ and the $\mathrm{L} 40^{\text {htphtprtrt }}$ line which contrast regarding the presence of the recently described dominant $H t P$ and the recessive $i t$ genes that confer resistance to Exserohilum turcicum. Five microsatellite markers revealed polymorphisms between the NIL and were considered candidate linked markers for the HtP resistance gene. Linkage was confirmed by bulked segregant sample (BSS) analysis of 32 susceptible and 34 resistant plants from a $B_{1} F_{1}$ population derived from the cross (L30 HtPHtPRtRt $\left.\times L 40^{\text {htphtprtrt }}\right) \times L 40^{\text {htphtprtrt }}$. The bnlg198 and dupssr25 markers, both located on maize chromosome $2 \mathrm{~L}$ (bin 2.08), were polymorphic between bulks. Linkage distances were estimated based on co-segregation data of the 32 susceptible plants and indicated distances of 28.7 centimorgans (cM) between HtP and bnlg198 and $23.5 \mathrm{cM}$ between HtP and dupssr25. The same set of susceptible plants was also genotyped with markers polymorphic between L30 HtPHtPRtRt and L40 htphtprtrt in order to find markers linked to the $r t$ gene. Marker bnlg197, from chromosome 3L (bin 3.06), was found linked to $r$ at a distance of $9.7 \mathrm{cM}$. This is the first report on the chromosomal locations of these newly described genes.
\end{abstract}

Key words: bulked segregant sample analysis, microsatellite markers, maize, near-isogenic lines, northern leaf blight.

Received: July 13, 2006; Accepted: November 6, 2006.

Helminthosporiosis is one of the main leaf diseases of maize and is caused by the fungus Exserohilum turcicum Leonard \& Suggs [Helminthosporium turcicum Pass.], which is the teleomorph of Setosphaeria turcica (Lutterell) Leonard \& Suggs (Frederiksen, 1991). Severe epidemics occur most frequently in Southern and Western Brazil, causing severe yield losses, which can exceed $50 \%$, as a consequence of extensive leaf damage during the grainfilling period (Raymundo and Hooker, 1981).

This disease can be controlled by deploying genes that confer either qualitative or quantitative resistance. Most of the qualitative resistance genes are dominant or partially dominant, such as the $\mathrm{Ht}_{1}, \mathrm{Ht}_{2}, \mathrm{Ht}_{3}, \mathrm{HtM}, \mathrm{HtN}$, and HtP genes (Gevers, 1975; Hooker 1961, 1963a, 1963b, 1975, 1977, 1978, 1981; Ogliari et al., 2005; Robbins and Warren, 1993), but there are also two examples of recessive genes (Carson, 1995; Ogliari et al., 2005). The resistance

Send correspondence to L.E.A.C. Av. Pádua Dias 11, Setor de Fitopatologia, Escola Superior de Agricultura Luiz de Queiroz, Universidade de São Paulo, 13418-900 Piracicaba, SP, Brazil. E-mail: leacamar@esalq.usp.br. genes $H t P$ and $r t$ were recently described by Ogliari et al. (2005). The dominant HtP confers resistance to all $E$. turcicum races described to date, whereas the recessive $r t$ gene confers resistance to races possessing the 1,2,3, and $\mathrm{N}$ virulence factors as well as the, as yet undetermined, $\mathrm{x}$ virulence factor (Ogliari et al., 2005). Both the HtP and $r t$ genes were identified in two elite maize lines (L30 ${ }^{\text {HtPHtPRtRt }}$ and L40 htphtprtrt) in a hybrid breeding program developed by Sementes Agroceres S/A (currently Monsanto do Brasil Ltda) through phenotypic analysis of $\mathrm{BC}_{1} \mathrm{~F}_{1}$ segregants (Ogliari et al., 2005).

Several qualitative genes and quantitative trait loci (QTL) controlling disease resistance have been mapped in maize, including E. turcicum resistance genes (Simcox and Bennetzen, 1993). For instance, the $H t_{1}$ gene is located on the long arm of chromosome 2, 168 centimorgans (cM) from the centromere (Bentolila et al., 1991). The two dominant genes $\mathrm{Ht}_{2}$ and $\mathrm{HtN}$, which are non-allelic and map $10 \mathrm{cM}$ from each other, are located on the long arm of chromosome 8 (Simcox and Bennetzen, 1993). Based upon reciprocal translocation mapping studies, a recessive gene 
appears to be located on the short arm of chromosome 1 near the centromere (Carson, 1995). However, until now the chromosomal locations of the HtP and $r t$ resistance genes mentioned above have not been determined.

Analyses of near-isogenic lines (NIL) and of bulked segregant samples (BSS) constitute two approaches commonly used to identify molecular markers linked to genes (Muehlbauer et al., 1988; Michelmore et al., 1991). In breeding programs, near-isogenic lines are easily obtained by backcrossing when a specific gene is introgressed from a donor line into a recurrent line. Thus, near-isogenic lines should be genetically identical over large portions of the genome, except for the segment containing the introgressed gene. Putative evidence of linkage between a marker and the target gene arises when the marker detects polymorphisms between near-isogenic lines. On the other hand, bulked segregant sample analysis involves comparisons between two-pooled DNA samples consisting of individual plants with extreme phenotypes identified in a segregating population. Evidence of linkage between a marker and the target gene arises when the marker is polymorphic between bulks. Thus, both strategies can be used concomitantly to identify putative markers linked to resistance genes. However, co-segregation analyses based on data from a segregating population is always required in order to confirm linkage and to estimate genetic distances.

The objective of the work described in this paper was to use near-isogenic lines and bulked segregant sample analyses to identify microsatellite (also called simple sequence repeats or SSRs) loci of known chromosomal locations linked to the HtP and $r t$ genes. Linkage was confirmed by co-segregation analyses between these genes and candidate markers using susceptible individuals of a $\mathrm{BC}_{1} \mathrm{~F}_{1}$ population. For this, the inbred resistant line L $30{ }^{H t P H t P R t R t}$ was generated after six backcrossing cycles between the recurrent L30 ${ }^{\text {htphtpRtRt }}$ susceptible line and the donor line L10 ${ }^{\text {HtPHtP }}$ followed by two self-pollinations $\left(\mathrm{BC}_{6} \mathrm{~S}_{2}\right)$ that brought the HtP locus to the homozygous state. The recurrent line was developed in central Brazil through selfpollinations and selections out of a synthetic composed of dent endosperm lines from the International Maize and Wheat Improvement Center (CIMMYT). The heterozygous stock for HtP was obtained from the cross $\mathrm{L} 30^{H t P H t P R t R t} \times \mathrm{L} 30^{\text {htphtpRtRt }}$. Finally, an $\mathrm{F}_{1}$ progeny from the cross L $30^{\text {HtPHtPRtRt }} \times \mathrm{L} 40^{\text {htphtprtrt }}$ and a $\mathrm{BC}_{1} \mathrm{~F}_{1}$ population from [(L30 $\left.{ }^{H t P H t P R t R t} \times \mathrm{L}^{\text {htphtprtrt }}\right) \times$ L40 ${ }^{\text {htphtprtrt }}$ ] were used both for bulked segregant sample and co-segregation analyses. The $\mathrm{BC}_{1} \mathrm{~F}_{1}$ plants were evaluated for lesion type using the score scale (decribed by Esteves MCF (1989). Master's Thesis, Escola Superior de Agricultura "Luiz de Queiróz", Universidade de São Paulo), where plants were considered resistant if they displayed chlorotic-necrotic lesions or did not display any symptoms at all and susceptible if they displayed olive green necrotic lesions (Ogliari et al., 2005). The inbred resistant line
L40 ${ }^{\text {htphtprtrt }}$ was developed in the southern sub-tropical region of Brazil by self-pollinations and selections from a synthetic produced by intercrosses between Brazilian commercial hybrids.

DNA was extracted according to Hoisington et al. (1994) and quantified by fluorimetry (DNA Quant200, Hoefer) and PCR amplifications of microsatellite loci were carried out using maize MapPairs primers (Research Genetics, USA). Reactions were carried out as described by Ogliari et al. (2000). Amplified fragments were separated in $3 \%(\mathrm{w} / \mathrm{v})$ agarose gels containing ethidium bromide at a concentration of $0.5 \mu \mathrm{g} \mathrm{mL}^{-1}$ of gel. Gels were run in $1 \mathrm{X}$ TBE buffer (0.09 $\mathrm{M}$ tris-borate and 2.0 mM EDTA, $\mathrm{pH}$ 8.3) at $80 \mathrm{~V}$ for 3 to $5 \mathrm{~h}$.

Lines L10 ${ }^{H t P H t P}, \quad$ L30 HtPHtPRtRt,$\quad$ L30 $0^{\text {htphtpRtRt }}$, L40 ${ }^{\text {htphtprtrt }}$ and the hybrid L30 ${ }^{\text {HtphtpRtRt }}$ were genotyped with 125 microsatellite loci chosen to represent the 10 maize chromosomes. Markers that were monomorphic between the donor parent (L10 ${ }^{H t P H t P}$ ) and the resistant line (L30 ${ }^{H t P H t P R t R t}$ ) but polymorphic between these two and the recurrent line (L30htphtpRtRt $)$ were considered potentially linked to the HtP locus. Such markers were further analyzed by bulked segregant sample analysis using individual plants from a segregating $\mathrm{BC}_{1} \mathrm{~F}_{1}$ population of 138 plants from the backcross [(L30 HtPHtPRtRt $\mathrm{x} \mathrm{L40}$ htphtprtrt $) \mathrm{x}$ L40 ${ }^{\text {htphtprtrt }}$ ] previously evaluated for resistance to race 123x of E. turcicum (Ogliari et al., 2005). Two DNA bulks from 34 resistant and 32 susceptible plants were genotyped with the candidate markers identified in the near-isogenic lines analysis. Linkage between $H t P$ and markers that revealed polymorphisms between bulks was tested in a co-segregation analysis as described below. Co-segregation between the $r t$ gene and polymorphic microsatellite markers between L30 HtpHtpRtRt and $\mathrm{L} 40^{\text {htphtprtrt }}$ was also tested in the same $\mathrm{BC}_{1} \mathrm{~F}_{1}$ population.

Co-segregation analyses between polymorphic markers and the HtP and $r t$ genes were carried out using the 32 plants from the susceptible bulk. Because $H t P$ is dominant and $r t$ is recessive (Ogliari et al., 2005), the genotype of all susceptible plants of the backcross [(L30 HtPHtPRtRt $\mathrm{x}$ L40 ${ }^{\text {htphtprtrt }}$ ) x L40 ${ }^{\text {htphtprtrt }}$ ] should be htphtpRtrt. Thus, for markers linked to HtP, the ratio between homozygotes and heterozygotes should differ by the chi-squared $\left(\chi^{2}\right)$ test from the 1:1 ratio expected for non-linkage, that is, an excess of homozygotes for marker genotypes should be detected in relation to heterozygous recombinants. The same reasoning applies to $r t$, except that in this case the heterozygotes should be in excess.

Assuming that all susceptible plants were homozygous recessive for HtP (htphtp) (Ogliari et al., 2005), the recombination frequency $\left(c_{1}\right)$ between the linked marker locus and $H t P$ relative to the susceptible plants can be estimated by $c_{1}=\mathrm{N}_{1} / \mathrm{N}_{\mathrm{s}}$, where $\mathrm{N}_{1}$ corresponds to the number of heterozygous recombinant plants for the marker locus and Ns to the total number of susceptible plants genotyped. 
Similarly, the recombination frequency $\left(\mathrm{c}_{1}\right)$ between the linked marker locus and $r t$ can be estimated by $\mathrm{c}_{1}=\mathrm{N}_{2} / \mathrm{N}_{\mathrm{s}}$, where $\mathrm{N}_{2}$ corresponds to the number of homozygous recombinant plants for the marker locus and $\mathrm{N}_{\mathrm{S}}$ to the total number of susceptible plants genotyped. For both genes, standard error estimates of $\mathrm{c}_{1}$ were given by $\mathrm{V}_{\mathrm{c}}{ }^{1 / 2}=\left\{\left[\mathrm{c}_{1}(1\right.\right.$ $\left.\left.\left.-\mathrm{c}_{1}\right)\right] / \mathrm{N}_{\mathrm{s}}\right\}^{1 / 2}$ (Liu, 1998) and the genetic distances were estimated by Haldane's mapping function (Haldane, 1919).

Sixty-one microsatellite markers were considered informative since they revealed polymorphism between the donor $\mathrm{L} 10^{H t P H t P}$ and the recurrent L30 $0^{\text {htphtpRtRt }}$ lines. Of these, however, 56 were monomorphic between L30 ${ }^{H t P H t P R t R t}$ and L30 ${ }^{\text {htphtpRtRt }}$, whereas the remaining five marker loci (the bnlg198 and dupssr25 from chromosome 2L (bin 2.08), ole2 (bin 5.02) and dupssr10 (bin 5.04) from chromosome $5 \mathrm{~S}$, and dupssr 15 from chromosome $6 \mathrm{~L}$ (bin 6.06)) were monomorphic between L $30^{H t P H t P R t R t}$ and L10 ${ }^{H t P H t P}$ but polymorphic between these two lines and L30 ${ }^{\text {htphtpRtRt }}$ and thus were considered potentially linked to the HtP locus. However, only two of these (bnlg198 and dupssr25) were confirmed by bulked segregant sample analysis as being linked to $H t P$ since they were polymorphic between the bulks, that is, the susceptible bulk amplified the same marker allele of the resistant line L $40^{\text {htphtprtrt }}$, whereas the resistant bulk amplified the alleles from both lines (L40 ${ }^{\text {htphtprtrt }}$ and L30 ${ }^{H t P H t P R t R t}$ ). These marker genotypes were expected, considering that $H t P$ confers dominant resistance and $r t$ confers recessive resistance (Ogliari et al., 2005). In this case, the genotype of all susceptible plants of the backcross [(L30 HtPHtPRtRt $\mathrm{x}$ L40 $\left.0^{\text {htphtprtrt }}\right) \mathrm{x}$ L40 htphtprtrt $]$ should be htphtpRtrt and the genotype for marker loci linked to $H t P$ should be homozygous in the sus- ceptible bulk for marker alleles of the resistant line L40 htphtprtrt.

Only seven bnlg198 heterozygotes and six dupssr 25 heterozygotes were found among the 32 susceptible plants of the backcross [(L30 $\left.{ }^{H t P H t P R t R t} \times \mathrm{L} 40^{\text {htphtprtrt }}\right) \mathrm{x}$ L40 $\left.0^{\text {htphtprtrt }}\right]$, which differ from the expected number $(p=0.01)$ of 16 plants in the case of non-linkage (Table 1$)$. Thus, the recombination frequency $\left(\mathrm{c}_{1}\right)$ between bnlg198 and $H t P$ was estimated to be $0.22 \pm 0.07\left[7 h t p M_{1} / h t p M_{2}\right.$ recombinants / $\left.32\left(h t p M_{1} / h t p M_{2}+h t p M_{2} / h t p M_{2}\right)\right]$ while that between dupssr25 and HtP was estimated to be $0.19 \pm 0.07\left[6 h t p L_{3} / h t p L_{4}\right.$ recombinants / $32\left(h t p L_{3} / h t p L_{4}\right.$ $\left.+h t p L_{4} / h t p L_{4}\right)$ ], whereas the recombination frequency between bnlg198 and dupssr 25 was $0.03 \pm 0.03$. These data indicate that $H t P$ is located on the long arm of chromosome 2 and is $28.7 \mathrm{cM}$ from bnlg 198 and $23.5 \mathrm{cM}$ from dupssr 25 (bin 2.08), the bnlg198 and dupssr 25 markers being $3.2 \mathrm{cM}$ from each other.

Twenty-four of the 125 microsatellite loci tested, were polymorphic between L $30^{H t P H t P R t R t}$ and L $40^{\text {htphtprtrt }}$, with bnlg197 being found to be linked to $r t$ since only three homozygotes for this marker were found. This differs $(p=0.01)$ from the 1:1 ratio expected (16 heterozygotes: 16 homozygotes) when there is no linkage. Assuming that the 32 susceptible plants were heterozygous for $r t$, the $\mathrm{c}_{1}$ value between bnlg197 and $r t$ was estimated to be $0.094 \pm 0.05$ [3 RtZ6/rtZ6 recombinant/32 (RtZ6/rtZ6 + RtZ5/rtZ6)]. These data indicate that $r t$ is located on the long arm of chromosome 3 (bin 3.06) distant $10.38 \mathrm{cM}$ of bnlg197.

In conventional linkage analysis, all markers that detect polymorphisms between the parental lines should be

Table 1 - Genetic linkage between $H t P$, bngl198 (M), and dupssr25 (L) and between $r t$ and bngl197 in the $\mathrm{BC}_{1} \mathrm{~F}_{1}$ population from [(L30 ${ }^{H t P H t P R t R t} \mathrm{x}$ $\mathrm{L} 40^{\text {htphtprtrt }}$ ) L L40 $0^{\text {htphtprtrt }}$.

\begin{tabular}{|c|c|c|c|c|c|}
\hline Linked loci & $\begin{array}{l}\text { Expected } \\
\text { frequency }\end{array}$ & $\begin{array}{l}\text { Observed } \\
\text { frequency }\end{array}$ & $\begin{array}{c}\text { Significant at } \mathrm{p}<0.01 \\
\text { by the } \chi^{2} \text { test }\end{array}$ & $\begin{array}{l}\text { Recombination } \\
\text { frequency estimate } \\
\qquad\left(\mathrm{C}_{1}, \%\right)\end{array}$ & $\begin{array}{l}\text { Standard error associated with the } \\
\text { estimated recombination frequency } \\
\qquad\left(\mathrm{V}_{\mathrm{c}}^{1 / 2}, \%\right)\end{array}$ \\
\hline \multicolumn{6}{|l|}{ HtP/bngl198 } \\
\hline$h t p M_{1} / h t p M_{2}$ & 16 & 7 & Yes & 21.87 & 7.31 \\
\hline$h t p M_{2} / h t p M_{2}$ & 16 & 25 & No & - & - \\
\hline \multicolumn{6}{|l|}{ HtP/dupssr25 } \\
\hline$h t p L_{3} / h t p L_{4}$ & 16 & 6 & Yes & 18.75 & 6.90 \\
\hline$h t p L_{4} / h t p L_{4}$ & 16 & 26 & No & - & - \\
\hline \multicolumn{6}{|l|}{$\mathrm{M} / \mathrm{L}$} \\
\hline$M_{1} L_{3} / M_{2} L_{4}$ & 8 & 6 & Yes & 3.12 & 3.07 \\
\hline$M_{1} L_{4} / M_{2} L_{4}$ & 8 & 1 & No & - & - \\
\hline$M_{2} L_{3} / M_{2} L_{4}$ & 8 & 0 & No & - & - \\
\hline$M_{2} L_{4} / M_{2} L_{4}$ & 8 & 25 & No & - & - \\
\hline \multicolumn{6}{|l|}{ rt/bng1197 } \\
\hline$R t Z_{\sigma} / r t Z_{6}$ & 16 & 3 & Yes & 9.37 & 5.15 \\
\hline$R t Z_{5} / r t Z_{6}$ & 16 & 29 & No & - & - \\
\hline
\end{tabular}


subsequently used for genotyping the entire segregating population. However, when near-isogenic lines or bulked segregant sample analyses are used, the number of genotyping reactions are greatly reduced, thus allowing a faster identification of linkage between markers at a reduced cost (Muehlbauer et al., 1988). The advantage of these techniques is improved if they are used together due to the low probability of false positives, i.e., informative markers that are polymorphic between near-isogenic lines or bulks but are not linked to the target gene (Michelmore et al., 1991).

In near-isogenic line analysis false positives can arise due to the retention of small segments of the genome of the donor parent in chromosomes of the converted line other than the chromosome carrying the target gene. False positives may also result from extensive retention of portions of donor parent-derived DNA in linkage blocks containing the gene of interest (linkage-drag). On the other hand, the probability of such false positives is smaller in bulked segregant sample analysis since only markers tightly linked to the target loci $(<30 \mathrm{cM})$ are detected, even if few $\left(\right.$ e.g. $10 \mathrm{~F}_{2}$ plants in each bulk) individual plants are used to construct the bulks (Michelmore et al., 1991). This probability is still smaller if a backcross population is used, because such crosses provide a good opportunity for crossing-over to occur between the desired gene(s) from the non-recurrent parent and any undesirable gene or genes linked to them (Wang and Peterson, 1994) thus resulting in a narrower "genetic window" around the target region compared to that occurring in $F_{2}$ populations. In our study, for instance, 5 markers were polymorphic between the isogenic lines for $H t P$, but only two of these were selected for co-segregation analysis because they also detected polymorphisms between bulks. As it turned out, these two were linked to $H t P$.

Co-segregation analysis using only susceptible plants confirmed linkage of the $H t P$ to the bnlg198 and dupssr 25 markers, both located on chromosome $2 \mathrm{~L}$ (bin 2.08). This shows that $H t P$ is located on the same chromosome as $H t_{1}$, a gene that was described more than 40 years ago (Patterson et al., 1965). These genes are distinct since they confer resistance to distinct races of E. turcicum (Ogliari et al., 2005) but our data did not allow us to confirm whether or not the $H t P$ and $H_{t}$ are alleles or if they are located at different loci. However, it is interesting to note that Hooker and Tsung (1980) suggested the existence of a second allele at the $H t_{1}$ locus, although they did not report any information regarding its phenotypic effects.

Since the backcross population used to map HtP also segregated for $r t$ and because these genes act independently and have opposite modes of gene action regarding resistance to E. turcicum, it was possible to find a marker linked to $r t$ as well using the same population. However, bulked segregant sample analysis could not be used in this case because no polymorphisms between bulks for markers linked to $r t$ would be expected, since the susceptible plants in the backcross population used in this study were heterozygous at this locus (i.e., htphtpRtrt) and the resistant plants could be either homo and/or heterozygous at this same locus (i.e., htphtprtrt, HtPhtpRtrt and/or HtPhtprtrt). Linkage between $r t$ and bnlg197 allowed locating this gene in the long arm of chromosome 3 (bin 3.06), where previous reports have indicated the existence of a quantitative loci (QTL) controlling resistance to E. turcicum (Freymark et al., 1993). As in the case of $H t P$, the results indicate either that $r t$ may be an allele of this locus with strong phenotypic effects or that it is linked to the QTL.

The identification of these two resistance genes (HtP and $r t$ ) is an important finding for defining genetic breeding strategies for maize to helminthosporiosis, because both the $H t P$ and $r t$ genes confer resistance to a wide spectrum of races of $E$. turcicum, even those that possess multiple virulence factors such as races 123x and 123N (Ogliari et al., 2005). In addition, qualitative resistance genes to $E$. turcicum have proved to be effective for long-term resistance to E. turcicum, with, for example, the $H_{t}$ gene providing protection against $E$. turcicum which lasted for 15 years after the release of cultivars containing this gene (Leath et al., 1990).

In Brazil, E. turcicum populations seems to be more diverse in terms of race composition, even so some studies observed a predominance of race 0 and the presence of some races capable of overcoming resistance conferred by the $\mathrm{Ht}_{1}$ gene (Gianasi et al., 1996). In this way, breeding strategies aiming to incorporate two or more widespectrum resistance genes into a single genotype could be an interesting strategy to control E. turcicum. Thus, both $\mathrm{L} 30^{\text {HtPHtPRTRt }}$ and L40 ${ }^{\text {htphtprtrt }}$ should be considered good sources of resistance to this pathogen in Brazil.

Even though our findings provided useful insights on the genetic basis of $H t P$ and $r t$ mediated resistance to $E$. turcicum, the linked markers found in our study are not adequate to be used in marker assisted selection programs due to their genetic distance. In order to do so, both allelism tests between $H_{t}$ and $H t P$ and saturation of the chromosomal regions around these loci with other markers should be accomplished.

\section{Acknowledgments}

The authors thank the Brazilian governmental agency CAPES for granting a scholarship to the first author, the São Paulo state agency FAPESP for financial support through grant 97/9531-4 and Sementes Agroceres S/A for providing the plant material.

\section{References}

Bentolila S, Guitton C, Bouvet N, Sailand A, Nykaza S and Freyssinet G (1991) Identification of RFLP marker tightly linked to the $H t_{1}$ gene in maize. Theor Appl Genet 82:393398. 
Carson ML (1995) A new gene in maize conferring the chlorotic halo reaction to infection by Exserohilum turcicum. Plant Dis 79:717-720.

Frederiksen RA (1991) Compedium of Sorghum Diseases. American Phytopathology Society, St. Paul, 82 pp.

Freymark PJ, Lee M, Woodman WL and Martison CA (1993) Quantitative and qualitative trait loci affecting host-plant response to Exserohilum turcicum in maize (Zea mays). Theor Appl Genet 87:537-544.

Gevers HO (1975) A new major gene for resistance to Helminthosporium turcicum leaf blight of maize. Plant Dis Rep 59:296-300.

Gianasi L, Castro HA and Silva HP (1996) Raças fisiológicas de Exserohilum turcicum identificadas em regiões produtoras de milho no Brasil, Safra 93/94. Summa Phytopathol 22:214-217.

Haldane JBS (1919) The combination of linkage values and the calculation of distances between the loci of linked factors. Journal of Genetics 8:299-309.

Hoisington D, Khairallah M and González de León D (1994) Laboratory Protocols: CIMMYT Applied Molecular Genetics Laboratory. 2nd edition. CIMMYT, México, $51 \mathrm{pp.}$

Hooker AL (1961) A new type of resistance in corn to Helminthosporium turcicum in seedling corn. Plant Dis Rep 45:780-781.

Hooker AL (1963a) Inheritance of chlorotic-lesion resistance to Helminthosporium turcicum in seedling corn. Phytopathology 53:660-662.

Hooker AL (1963b) Monogenic resistance of Zea mays L. to Helminthosporium turcicum. Crop Sci 3:381-383.

Hooker AL (1975) Helminthosporium turcicum as a pathogen of corn. Reporter Tottori Mycology Institute 12:115-125.

Hooker AL (1977) A second major gene locus in corn for chlorotic-lesion resistance to Helminthosporium turcicum. Crop Sci 17:132-135.

Hooker AL (1978) Additional sources of monogenic resistance in corn to Helminthosporium turcicum. Crop Sci 17:132-135.

Hooker AL (1981) Resistance to Helminthosporium turcicum from Tripsacum floridanum incorporated into corn. Maize Genet Cooperation News Lett 55:87-88.

Hooker AL and Tsung YK (1980) Relationship of dominant genes in corn for chlorotic lesion resistance to Helminthosporium turcicum. Plant Dis 64:387-388.
Leath S, Thakur RP and Leonard KJ (1990) Variation in expression of monogenic resistance in corn to Exserohjilum turcicum race 3 under different temperature and light regimes. Phytopathology 80:309-313.

Liu BH (1998). Statistical Genomics: Linkage, Mapping, and QTL Analysis. 2nd edition. CRC Press LLC, Boca Raton, $611 \mathrm{pp}$.

Michelmore RW, Paran I and Kesseli RV (1991) Identification of markers linked to disease-resistance genes by bulked segregant analysis: A rapid method to detect markers in specific genomic regions by using segregating populations. Proc Natl Acad Sci USA 86:9828-9832.

Muehlbauer GL, Specht JE, Thomas-Compton MA, Staswick PE and Bernard RA (1988) Near isogenic lines - A potential resource in the integration of conventional and molecular marker linkage maps. Crop Sci 28:729-735.

Ogliari JB, Boscariol RL and Camargo LEA (2000) Optimization of PCR amplification of maize microssatellite loci. Genet Mol Biol 23:393-398.

Ogliari JB, Guimarães MA, Geraldi IO and Camargo LEA (2005) New resistance genes in the Zea mays L. - Exserohilum tucicum pathosystem. Genet Mol Biol 28:435-439.

Patterson EB, Hooker AL and Yates, DE (1965) Location of Ht in the long arm of chromosome 2. Maize Genet Cooperation News Lett 39:86-87.

Raymundo AD and Hooker, AL (1981) Measuring the relationship between northern corn leaf blight and yield losses. Plant Dis 65:325-327.

Robbins WA and Warren HL (1993) Inheritance of resistance to Exserohilum turcicum in PI 209135, "Mayorbela" variety of mayze. Maydica 38:209-213.

Senior ML and Heun M (1993) Mapping maize microsatellites and polymerase chain reaction confirmation of the targeted repeats using a CT primer. Genome 36:884-889.

Simcox KD and Bennetzen JL (1993) The use of molecular markers to study Setosphaeria turcica resistance in maize. Phytopathology 83:1326-1330.

Wang GL and Paterson AH (1994) Assessment of DNA pooling strategies for mapping of QTLs. Theor Appl Genet 88:355361.

Associate Editor: Everaldo Gonçalves de Barros 